disturbances of the normal flora, and promotion of bacterial resistance. Agreement was almost complete on a question about antibiotic penetration of operative wounds and the time taken to achieve it. Penicillin and ampicillin are said to reach peak concentrations in wound fluid within one hour and cephalosporins within two but clindamycin and erythromycin may take five hours. All were agreed that to start administration 24 hours beforehand was unnecessary and that the object should be to achieve maximum concentrations at the time of operation when bacterial implantation may occur. Many surgeons follow the practice of giving three doses, one immediately before and the others 5 and 12 hours after operation, as originally advocated by Polk and Lopez-Mayor. ${ }^{2}$

In basically clean surgery it was agreed that antibiotics were usually unnecessary. "Clean-contaminated" operations such as gastrectomy, cholecystectomy for acute cholecystitis, and elective resection of the colon were the subject of more varied replies. A distinction may be drawn between peptic ulcer and cancer as the indication for gastrectomy, for the latter is said to carry a greater risk of sepsis. For operations on the colon there were two votes in favour of cephaloridine and one for intravenous penicillin and tetracycline continued for three to four days. Only one reply referred to orally administered antibacterials, which were approved, together with mechanical bowel preparation; surprisingly, this much debated methodwhich has more strong opponents than advocates in the United States-was not further discussed. Vascular and orthopaedic procedures in which a foreign body was implanted were discussed on the basis that foreign bodies markedly reduced local tissue antibacterial capability, and that infection, though uncommon, could have disastrous effects. The only positive recommendation made was based on the fact that infection was usually staphylococcal, and advocated two large doses of oxacillin or two to five days' treatment with cloxacillin. This is a subject which, particularly as it concerns cardiac surgery, might well have been more fully discussed. One vital point was made: that though an antibiotic may dispose of bacteria implanted at operation others may reach the site afterwards by way of arterial lines, central venous lines, and endotracheal tubes, and treatment should therefore be continued until these are removed.

No-one liked peritoneal irrigation with antibiotics; one suggestion was that the mechanical effects of lavage, as with saline, were what mattered, and another that the peritoneum, with its profusion of active phagocytes, could look after itself. Wounds were thought a different matter, and one of the surgeons made a practice of irrigating wounds in immunosuppressed patients with a solution of kanamycin. For surgical closure of a peptic ulcer perforated four hours earlier the usual treatment with cephaloridine was recommended in one reply and penicillin with tetracycline in another. A question about the treatment of apparently anaerobic infections in which a film shows pleomorphic Gram-negative bacilli was prefaced by the remark that more anaerobic infections were being seen nowadays. An alternative possibility is that the number of such infections is unchanged, but laboratories have become more interested in them and diligent in looking for them. Two replies advocated clindamycin combined with penicillin and gentamicin if the flora were mixed and two chloramphenicol. There was no mention of metronidazole, the activity of which against Bacteroides spp. shows great promise. $^{34}$

Two other questions concerned the possibility of superinfection by organisms resistant to the antibiotic used, a point taken up by the editor in his final remarks, which emphasized that "one must be able to predict with some accuracy the type of bacteria one wishes to inhibit," since it was truly impossible to bar the entire bacterial world. But the question of how much of it is to be barred remains; should all treatment be as narrowly directed as he implies, or is there any place left for broad spectrum coverage ? Perhaps the most disputable of all the recommendations made is the use of tetracycline, which has fallen out of favour because both of frequent resistance to it in many bacterial species and of its disturbing effect on the normal bowel flora.

${ }^{1}$ Hunt, T. K., et al., Archives of Surgery, 1975, 110, 148.

${ }^{2}$ Polk, H. C., and Lopez-Mayor, J. F., Surgery, 1969, 66, 97.

${ }^{3}$ Nastro, L. J., and Finegold, S. M., Fournal of Infectious Diseases, 1972, 126, 104.

4 Whelan, J. P. F., and Hale, J. H., fournal of Clinical Pathology, 1973, 26, 393.

\section{Classification of Lymphomata}

Until quite recently the malignant lymphomata were classified into four groups: reticulum-cell sarcoma, lymphosarcoma, giant follicular lymphoma, and Hodgkin's disease. Twenty years ago Rappaport ${ }^{12}$ had noted that follicular lymphoma was not a specific entity but merely the expression of a nodular disposition of lymphomatous tumour tissue as compared with the rather more frequent, and prognostically worse, diffuse distribution. Soon afterwards Hodgkin's disease was separated off and classified on histological criteria by Lukes. ${ }^{34}$ Doubt was cast on the nature of reticulum-cell sarcoma: its cell of origin was thought to be much more frequently the histiocyte than the reticular cell of lymphoid tissue. Rappaport consequently proposed a new classification for the lymphomata, excluding Hodgkin's disease, based on three features: the pattern of the tumour (nodular or diffuse), the cellular type (lymphocytic, histiocytic, or mixed lymphocytic-histiocytic), and the degree of differentiation of the cell (well differentiated, poorly differentiated, and undifferentiated).

Lymphocytes fall into two categories, the $\mathrm{T}$ cell, which is thymic dependent, and the $\mathrm{B}$ cell, which is thymic independent. In fowls B cells depend on the bursa of Fabricius, but the mammalian equivalent of this organ is unknown. $T$ cells lead to spontaneous rosette formation of sheep erythrocytes, while B cells have specific receptor sites for heavy and light chains of immunoglobulin, and these tests can be used to distinguish the two types of lymphocytes. Histiocytes contain a non-specific esterase which can be detected in tissue imprints or frozen sections, and they can thereby be differentiated from lymphocytes. So the lymphomata can now be classified functionally according to their cell of origin.

A recent supplement to the British fournal of Cancer records papers presented at the Symposium on Non-Hodgkin's Lymphomata held at London in $1973 .{ }^{5}$ Among the many valuable contributions is the paper by Lukes and Collins, ${ }^{6}$ who explore new approaches to the classification of the lymphomata. They define five major cell groups: the $U$ cell (undefined and without specific markers), the T cell, the B cell, the histiocyte (macrophage), and unclassifiable (for those incapable of specific cytological classification).

There are no firmly established T-cell lymphomata, but the Sézary syndrome, mycosis fungoides, and a variety of acute lymphocytic leukaemia with a mediastinal mass probably fall into this category, as does also Hodgkin's disease. Most 
lymphomata arise from B cells, which in turn develop in the centres of the lymphoid follicles found typically at the periphery of the lymph node. Lukes and Collins trace a transformation of small lymphocytes in the follicular centre through a possible plasmacytoid stage to a phase of nuclear cleavage which culminates in the development of a non-cleaved cell with much pyroninophilic cytoplasm and a number of nucleoli. These follicular centre cell (F.C.C.) types are classified as small cleaved, large cleaved, small non-cleaved, and large non-cleaved. The last resembles a fully transformed lymphocyte, and is called an immunoblast when it leaves the follicles and enters the interfollicular tissue.

On the basis of this development of the B cell, seven types of lymphomata can be recognized: chronic lymphatic leukaemia from the small lymphocyte, the closely related macroglobulinaemia described by Waldenström from the plasmacytoid variant of the small lymphocyte, lymphomata from each of the four types of F.C.C., of which the cleaved cells show the greater tendency to nodular dispositions and the better prognosis, and finally the immunoblastic sarcoma, which closely resembles a lymphoma arising from the large non-cleaved cell. It may occur as a complication of chronic abnormal immune states, in Sjögren's syndrome, in patients on immunosuppressive therapy, or in the aged. Histiocytic lymphomata are rare, for nearly all the cells that were previously called histiocytes (or reticular cells) are now shown to be transformed lymphocytes.

This classification, though complicated, throws much light on the histogenesis of the lymphomata, for it combines functional studies with the purely cytological description that was the basis of previous classifications. It elucidates the nodular and diffuse disposition of lymphoma cells, and also puts into clearer perspective the condition previously called reticulum-cell sarcoma.

1 Rappaport, H., Winter, W. J., and Hicks, E. B., Cancer, 1956, 9, 792.

2 Rappaport, H., Tumours of the Haematopoietic System, p. 91. Washington, D.C.: Armed Forces Institute of Pathology, 1966.

${ }^{3}$ Lukes, R. J., Butler, J. J., and Hicks, E. B., Cancer (Philadelphia), 1966, 19, 317.

${ }^{4}$ Lukes, R. J., Cancer Research, 1966, 26, 1311.

5 Symposium on Non-Hodgkin's Lymphomata, British fournal of Cancer, 1975, 31, Supplement No. II.

${ }^{6}$ Lukes, R. J., and Collins, R. D., British Fournal of Cancer, 1975, 31, Supplement No. II, 1.

\section{Diabetic Nephropathy}

Diabetic nephropathy can be a dangerous disorder with a variable and unpredictable course. However, since new treatments by dialysis and transplantation are still being developed, more detailed knowledge of the natural history of the disorder is needed.

Proteinuria is the clinical hallmark of diabetic nephropathy and the simplest guide to its prognosis. It becomes increasingly common as duration of diabetes increases, ${ }^{1}$ and in young diabetics most deaths occur in patients with proteinuria. ${ }^{2}$ It has been estimated that $77 \%$ of young patients will be dead 10 years after the appearance of proteinuria. ${ }^{3}$ Prognosis is worst in those with heavy proteinuria, ${ }^{4}$ and the nephrotic syndrome therefore represents a very advanced stage of the disease. ${ }^{5}$ Patients with diabetic nephropathy have a greatly increased risk of atheroma, and this is intensified by both increasing age and duration of diabetes. ${ }^{6}$ Death is mainly due to uraemia in younger patients and to myocardial infarction in the older ones. ${ }^{7}$

Many renal biopsy studies in diabetics have attempted to evaluate the disease's severity, and sometimes serial biopsies have been used to follow its course. ${ }^{489}$ There is some correlation between histological changes, proteinuria, and renal function, especially with diffuse glomerulosclerosis. ${ }^{8}$ The most recent and extensive study of serial biopsy specimens comes from Japan, ${ }^{10}$ where the results of 23 renal biopsies were reviewed at further biopsy (or in two cases at necropsy) two to eight years later. Progression of the renal lesion was observed in 13, but there had been no change in 10. It is generally agreed that lesions once established never regress, but-as in the case of retinopathy-they do not necessarily progress. Advanced lesions seem to deteriorate more rapidly than early ones, and this is in keeping with clinical observations. The course of the disease is not reliably predictable from histological appearances, and occasionally even the more severe lesions may not change over several years. Nodular lesions and the fibrinoid cap both forecast a bad outlook, but even then exceptions do occur. It is, therefore, rarely helpful to undertake a renal biopsy in these patients unless the diagnosis is in serious doubt.

The factors causing diabetic nephropathy or those determining its progression are still unknown. There is no convincing evidence that poor control causes diabetic nephropathy or in any way alters its course. The recent Japanese biopsy study attempted to relate control to the progression of biopsy appearances, but in fact it showed how difficult interpretation can be, since the different groups of patients were not really comparable. Experimentally there is evidence that diabetic glomerulosclerosis may actually regress if the kidneys are transplanted into non-diabetic animals, ${ }^{11}$ and after islet transplantation, ${ }^{12}$ but such studies are a long way from reality. On the other hand there is as yet no evidence that normal kidneys transplanted into diabetics develop specific changes of diabetic nephropathy, ${ }^{13}$ but follow-up periods have been relatively short.

Most physicians believe that these patients should receive the best possible diabetic control-often difficult in renal failure-but it remains disappointing that there is no scientific evidence of a reward for doing so. Control of hypertension, which occurs late in the course of the disease, is obviously desirable, but the effect on prognosis is unknown. Hyperlipidaemia is common in diabetic nephropathy patients ${ }^{8}$ and no doubt contributes to the atheroma to which they are particularly susceptible, but at present one cannot be sure whether its control is beneficial. Growth hormone excess ${ }^{14}$ is often blamed for diabetic complications, but again this is controversial and there is no good evidence that its control improves diabetic nephropathy. Hypophysectomy actually causes glomerular filtration to fall. ${ }^{15}$ Somatostatin is still an experimental tool which decreases growth hormone secretion, ${ }^{16}$ and it will be many years before its clinical effects can be evaluated.

Once renal failure is established in diabetic nephropathy a downhill course is certain and survival is usually less than two years. ${ }^{3}$ Whether at this stage either the quality of life or its duration can be improved is still controversial. Chronic dialysis patients run a stormy course, and survival figures are poor when compared to non-diabetics. ${ }^{17} 18$ Renal transplantation may hold out better prospects, ${ }^{13}$ but even in these patients cardiovascular disease takes a heavy toll. The trend to perform transplantation at an earlier stage of the disease makes it more important than ever to understand the natural course of the disease and to try to discover any methods by which to influence it. 